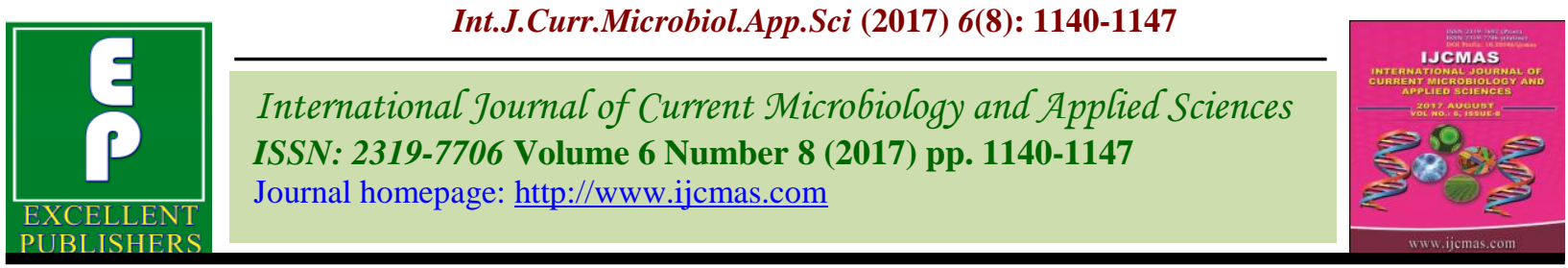

Original Research Article

https://doi.org/10.20546/ijcmas.2017.608.141

\title{
Determination of Minimum Inhibitory Concentration of Liposomes: A Novel Method
}

\author{
Atulya Manuel ${ }^{1 *}$ and Nabeel Abdulrahman ${ }^{2}$ \\ ${ }^{1}$ CCS National Institute of Animal Health, Baghpat, Uttar Pradesh, India \\ ${ }^{2}$ College of Pharmacy, Qatar University, Doha, Qatar \\ *Corresponding author
}

A B S T R A C T

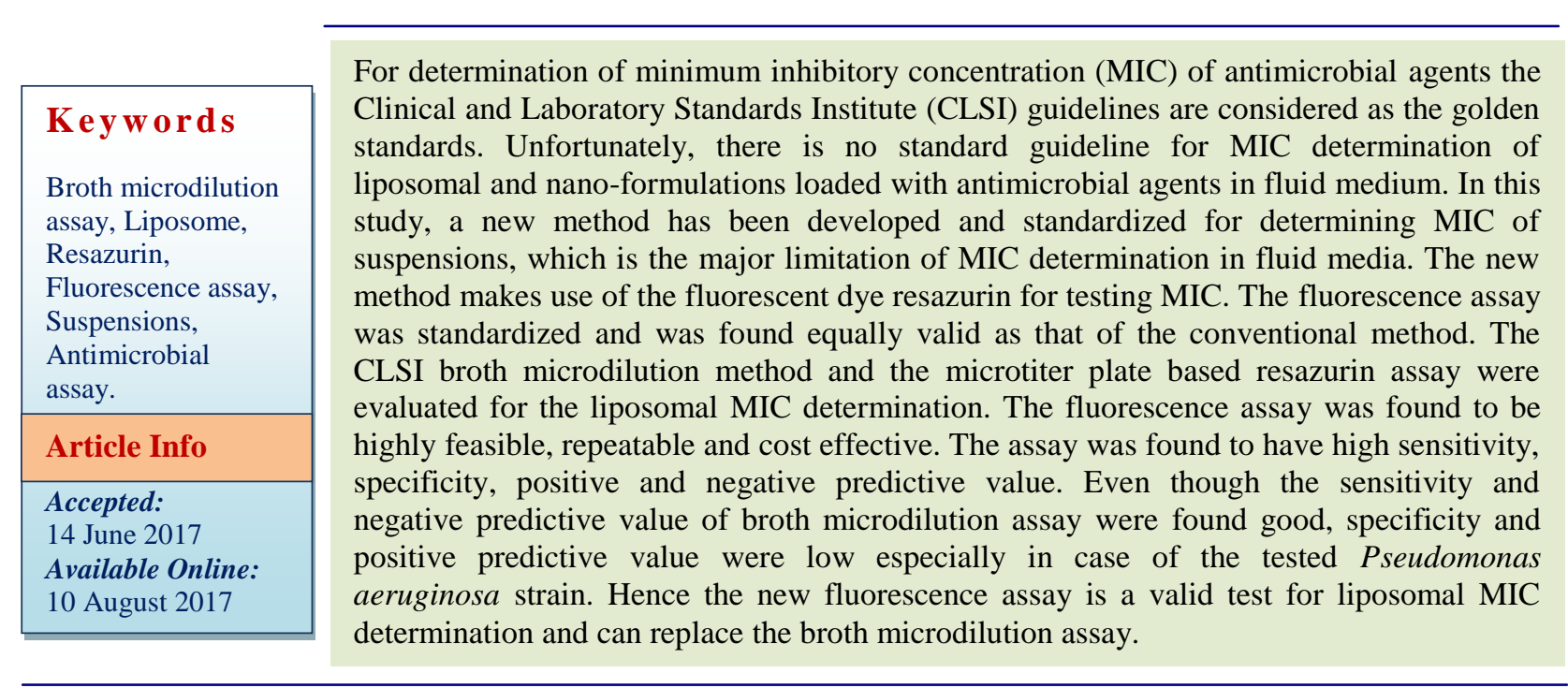

\section{Introduction}

The rate of development of antibiotic resistance by microorganisms is pretty high compared to the discovery of new antibiotics. Therefore new formulations have come up in order to avoid resistance, either through increasing the availability or penetration of the already existing drugs(Arias, 2013). Delivery of antimicrobials or other compounds in the form of liposome is gaining more attention (Chattopadhyay, 2013, Rathore et al., 2011, Alhariri et al., 2013). At present there are no standard broth microdilution methods for determination of minimum inhibitory concentration (MIC) of liposomal drugs, coloured or opaque compounds as well as liquids with suspended particles having antimicrobial activity.

In such situations methods that depend upon optical density are not reliable and fluorescent methods can be performed with more accuracy. Usually the MICs of drug entrapped liposomes are tested by broth microdilution assay of CLSI (formerly NCCLS) (Mugabe et 
al., 2006, Halwani et al., 2007, Institute, 2012) or by agar dilution method (Omri et al., 1995). Liposome suspension loaded with drugs is normally milky in nature. The present study discusses a novel fluorescent method we developed, standardized and evaluated for determination of the MIC of turbid solutions and suspensions. The validation study of fluorescent assay against microdilution method was also carried out.

Resazurin, used in this assay is a blue compound that can be reduced to resorufin which is pink coloured. Resorufin is a fluorescent compound and this will get further reduced to non-fluorescent hydroresorufin. This reduction of resazurin to resorufin is carried out by the metabolically active cells. This assay measures the live cells which are metabolically active. In the conventional broth microdilution assay, the optical density (OD) of drug dilutions, inoculated with microorganisms after sufficient incubation is taken into consideration, which correlates with the most metabolically active cells in the mid log phase of growth. The particles present in the liposomes and other particulate solutions interfere with the OD measurement and produce an erratic result. Resazurin method is more reliable and realistic as it depends on metabolic activity of cells rather than OD of particulate matter present in the samples.

\section{Materials and Methods}

\section{Antimicrobials and microorganisms used in the study}

Control strains used for antimicrobial susceptibility testing were used for testing the MIC by various methods. These cultures were procured from Microbial Type Culture Collection and Gene Bank (MTCC), Chandigarh, India and National Collection of Industrial Microorganisms (NCIM), Pune,
India. The strains used and their equivalent numbers are Staphylococcus aureus subsp. au MTCC 96 (ATCC 9144) and MTCC 1430 (ATCC 12600), Escherichia coli MTCC 739 (ATCC 10536) and Pseudomonas aeruginosa MTCC 5029 (ATCC 27853). Cirprofloxacin hydrochloride (Cipro) was obtained as a gift sample from Alembic Ltd., Vadodara, India. All the chemicals and reagents used were of analytical grade.

\section{Preparation of antimicrobial loaded liposomes}

Ciprofloxacin encapsulated liposomes (CL) were prepared by lipid hydration method (Bangham et al., 1965) and standardised. Soya phosphatidylcholine: cholesterol was taken in 4:1 ratio and dissolved in a solvent mixture of chloroform and methanol in a volume ratio of $2: 1$. This was dried in a rotary evaporator (Butchirota vapor R-215, Switzerland), at constant temperature $\left(30^{\circ} \mathrm{C}\right)$ under reduced pressure at 60rpm. The film formed on the round bottom flask was kept for overnight drying in a vacuum desiccator. Antibiotic solution prepared in milliQ water was used for hydration of lipids. The antibiotic solution was added gently through the sides and the round bottom flask and it was kept in Rotek -Griffin flask shaker for 30minutes for mixing.

This was sonicated using a probe for 10 minutes at $60 \%$ amplitude with $60 \mathrm{sec}$ on and $30 \mathrm{sec}$ off followed by separation and removal of the unentrapped drug by centrifugation at 20,000 rpm for 30minutes at $4^{\circ} \mathrm{C}$ and the pellet was resuspended in equal amount of milli Q water as that of the initial solvent. Liposomes were filter sterilized $(0.22 \mu$ filter $)$ and was used for further studies. Three sets of liposomes were prepared and performed the study for testing the repeatability of the experiment. 


\section{Physical characterisation of antimicrobial loaded liposomes}

The mean particle size, poly dispersity index and zeta potential of the antibiotic entrapped liposomes were determined using Malvern Nano Zetasizer (Malvern Instruments Ltd. UK.) after diluting the liposome preparation with milliQ water. The data acquisition and analysis was performed using the software Malvern DTS v.5.00. The particle size and surface morphology were examined by Scanning electron microscopy (ZEISS EVO 18 Special Edition). The percentage of encapsulation was determined (Cheow and Hadinoto, 2011).

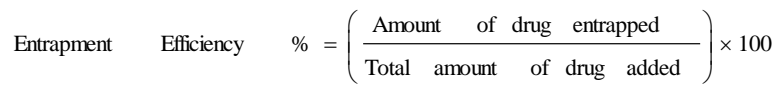

The amount of encapsulated drug was calculated by subtracting the drug amount present in the supernatant after centrifugation from the initial amount of drug used. The drug concentration was determined using UVVis spectrophotometer (Shimadsu, Japan).

Broth microdilution assay for determination of MIC of antimicrobial solution

The MIC of ciprofloxacin against different test organisms was determined by broth microdilution assay (Institute, 2012).

\section{Fluorescence Broth Microdilution Assay (FBMA) for determination of MIC of antimicrobial solution}

Bacterial inoculum was prepared by picking up five to six colonies and inoculating $4 \mathrm{~mL}$ of Mueller Hinton broth. After overnight incubation at $35 \pm 2^{\circ} \mathrm{C}$, a semi-confluent growth was obtained. Direct colony suspension method was practiced for Staphylococcus aureus inoculum preparation.
The turbidity was adjusted to $0.5 \mathrm{McFarland}$ standard. Hundred microliter of Mueller Hinton broth was added to all the wells except the negative control wells. $100 \mu \mathrm{l}$ of antibiotic solution was added to the first well of the raw and serially dilutions were made till the last well and $100 \mu 1$ was discarded from the last well. $10 \mu 1$ of inoculum was added to all the wells except negative control. The positive control wells had inoculated broth and negative wells had broth alone. The plates were incubated for 16 to $20 \mathrm{hrs}$ at $35 \pm 2^{\circ} \mathrm{C}$. All the tests were done in triplicates. The broad spectrum antibiotic Ciprofloxacin was chosen for testing. $0.02 \%$ of ciprofloxacin stock solution was made. Concentration of antibiotic in the first well was $100 \mu \mathrm{g} / \mathrm{mL}$.

After incubation the absorbance was measured at $625 \mathrm{~nm}$. In order to take the readings, the negative wells should be clear and positive wells should have a button of growth more than $2 \mathrm{~mm}$ should be present, indicating adequate growth in the MIC panel.

The same plate after absorbance reading was subjected to fluorescence assay. Standard stock solution of resazurin sodium was prepared by dissolving $270 \mathrm{mg}$ of the salt in $40 \mathrm{~mL}$ sterile double distilled water. Aliquotes were made in small Eppendorf tubes and stored at $-20^{\circ} \mathrm{C}$. The assay was performed using fluorescent microtitre plate raeder, FLX 800 (Biotek). The resazurin working solutions of $0.08,0.04,0.02$ and $0.01 \mathrm{mg} / \mathrm{mL}$ were prepared in cation adjusted Mueller-Hinton broth. After adding $100 \mathrm{~mL}$ of resazurin working solution fluorescence ( $\lambda$ ex: $560 \mathrm{~nm}$ and $\lambda \mathrm{em}: 590 \mathrm{~nm}$ ) was measured at $37^{\circ} \mathrm{C}$ for two hours. The readings were taken for two hours at 5minutes interval. Once the readings are over, the standard curve was constructed. The results were analyzed using Gen 5.106 software supplied along with the instrument. Presence of live cells was indicated by a peak in the plot between the fluorescence intensity and the time. The results of the flurescence 
assay were compared with the broth microdilution assay of CLSI for validation.

Comparison of FBMA for MIC of antimicrobial agents with Broth Microdilution Assay for validation

FBMA for MIC of antimicrobial agents was compared and validated against Broth Microdilution Assay using statistical analysis.

The various components of validity such as sensitivity, specificity, predictive value of a positive result and predictive value of a negative result were determined.

\section{Determination of MIC of liposomes using Broth Microdilution Assay and FBMA}

The assays were performed in 96 well micro titer plate as described above. $100 \mu \mathrm{l}$ of liposome was added instead of antibiotic solution, to the first well and serial dilutions were made.

After incubation the absorbance was taken in case of broth microdilution method and the same plates were subjected to fluorescence assay after adding resazurin as described above.

\section{Statistical analysis}

The FBMA for MIC of antimicrobial agents as well as for liposomes were validated against the broth microdilution method of CLSI which is the gold standard. The various components of validity such as sensitivity, specificity, predictive value of a positive result and predictive value of a negative result were determined. Each well was considered as a test and the formulae followed were as follows.

Sensitivity $=\frac{a}{(a+c)} \times 100$
Specificity $=\frac{d}{(b+d)} \times 100$

Positive predictive value $=\frac{a}{(a+b)} \times 100$

Negative predictive value $=\frac{d}{(c+d)} \times 100$

Where, (a) True positive, (b) False positive, (c) False negative, (d) True negative

\section{Results and Discussion}

\section{Liposome characterization}

The mean particle size was found to be 113.7 $\pm 1.65 \mathrm{~nm}$ with a poly dispersity index of $0.179 \pm .06$ and an average zeta potential of $-23.8 \pm 3.13 \mathrm{mV}$. The size quality report was found to meet the quality criteria. The scanning electron micrograph of the liposomes is given in figure 3 . The average percentage of entrapment efficiency was $79.22 \pm 1.96$ (Figs. 1 and 2).

Determination of MIC antimicrobial solution by broth microdilution assay

The MIC values and absorbance readings of the positive and negative control for MIC are given in table 1 .

Comparison and Validation of FBMA and broth microdilution assay for MIC of antimicrobial solution

When resazurin solution was added to the well, the live cells reduced the dye within $2 \mathrm{hrs}$ period in case of all the tested organisms. The standard curves were drawn using the fluorescence readings. Both the $S$. aureus strains reduced the dye and the fluorescence peaks were formed within 15 minutes Therefore immediately after adding the dye solution the reading has to be started. It is advisable to set the machine at $37^{\circ} \mathrm{C}$ and 
make a protocol prior to the addition the dye. Readings are taken in five minutes interval.

E. coli strain attained the peak fluorescence by 15 minutes and then goes down. $P$. aeruginosa fluorescence reached the peak value after half an hour. Even though it is declining subsequently, the reduction is not a steep one.

The well showing MIC can easily be detected by viewing the standard curve. The negative wells as well as the wells without any bacterial growth will give a horizontal line.

The last well showing the horizontal line was taken as the well with minimum inhibitory concentration of the antibiotic. Both the assays gave similar result for all the strains. The sensitivity, specificity, positive predictive value and negative predictive value were found to be 100 and the fluorescent method was found to be equally valid as of broth microdilution assay for MIC testing of antimicrobial solutions against planktonic bacteria.

\section{Validation study of liposomal MIC determination by FBMA against broth microdilution method}

FBMA and broth microdilution assay were compared for testing the MIC of antimicrobial encapsulated liposomal formulation. The number of true positive, false positive, false negative and true negative wells was calculated and is given in table 2 . The results were consistent when the tests were repeated. The test results were further confirmed by pour plate method. None of the negative wells gave growth and all the positive wells gave bacterial colonies.

Table.1 The MIC readings and control values

\begin{tabular}{|l|l|l|l|}
\hline Microorganism & MIC in $\mu \mathrm{g} / \mathrm{mL}$ & Positive control & OD $_{600}$ \\
\hline S. aureusMTCC96 & $<0.04$ & S. aureusMTCC96 & 1.065 \\
\hline S. aureus 1430 & 0.78 & S. aureus 1430 & 0.755 \\
\hline E.coli 739 & $<0.04$ & E.coli 739 & 1.0 \\
\hline P. aeruginosa 5029 & 0.78 & P. aeruginosa 5029 & 1.2 \\
\hline & & Negative control & 0.073 \\
\hline
\end{tabular}

Table.2 Different categories of wells obtained in FBMA and broth micro-dilution assay

\begin{tabular}{|c|c|c|c|c|c|c|c|c|}
\hline Categories & \multicolumn{4}{|c|}{ FBMA } & \multicolumn{4}{c|}{ Broth microdilution assay } \\
\cline { 2 - 9 } & $\begin{array}{c}\text { S.aureus } \\
\text { MTCC } \\
96\end{array}$ & $\begin{array}{c}\text { S.aureus } \\
\text { MTCC } \\
1430\end{array}$ & $\begin{array}{c}\text { E.coli } \\
\text { MTCC } \\
739\end{array}$ & $\begin{array}{c}\text { P.aeruginosa } \\
\text { MTCC 5029 }\end{array}$ & $\begin{array}{c}\text { S.aureus } \\
\text { MTCC 96 }\end{array}$ & $\begin{array}{c}\text { S.aureus } \\
\text { MTCC } \\
1430\end{array}$ & $\begin{array}{c}\text { E.coli } \\
\text { MTCC } \\
739\end{array}$ & $\begin{array}{c}\text { P.aeruginosa } \\
\text { MTCC 5029 }\end{array}$ \\
\hline TPW & 27 & 6 & 30 & 3 & 27 & 6 & 30 & 3 \\
\hline FPW & 0 & 0 & 0 & 0 & 3 & 3 & 6 & 33 \\
\hline FNW & 0 & 0 & 0 & 0 & 0 & 3 & 0 & 0 \\
\hline TNW & 45 & 30 & 42 & 33 & 45 & 30 & 42 & 33 \\
\hline
\end{tabular}

TPW: true positive wells, FPW: false positive wells, FNW: false negative wells, TNW: true negative wells 
Table.3 Results of validation study of MIC testing using FBMA and broth micro-dilution method

\begin{tabular}{|c|c|c|c|c|c|c|c|c|}
\hline \multirow[b]{2}{*}{$\begin{array}{l}\text { Validation } \\
\text { parameters }\end{array}$} & \multicolumn{4}{|l|}{ FBMA } & \multicolumn{4}{|c|}{ Broth microdilution assay } \\
\hline & $\begin{array}{l}\text { S.aureus } \\
\text { MTCC } \\
96\end{array}$ & $\begin{array}{l}\text { S.aureus } \\
\text { MTCC } \\
1430\end{array}$ & $\begin{array}{l}\text { E.coli } \\
\text { MTCC } \\
739\end{array}$ & $\begin{array}{l}\text { P.aeruginosa } \\
\text { MTCC5029 }\end{array}$ & $\begin{array}{l}\text { S.aureus } \\
\text { MTCC } \\
96\end{array}$ & $\begin{array}{l}\text { S.aureus } \\
\text { MTCC } \\
1430\end{array}$ & $\begin{array}{l}\text { E.coli } \\
\text { MTCC } \\
739\end{array}$ & $\begin{array}{l}\text { P.aeruginosa } \\
\text { MTCC5029 }\end{array}$ \\
\hline Sensitivity & 100 & 100 & 100 & 100 & 100 & 66.67 & 100 & 100 \\
\hline Specificity & 100 & 100 & 100 & 100 & 93.75 & 90.9 & 87.5 & 50 \\
\hline PPV & 100 & 100 & 100 & 100 & 90 & 66.67 & 83.33 & 8.33 \\
\hline NPV & 100 & 100 & 100 & 100 & 100 & 90.9 & 100 & 100 \\
\hline
\end{tabular}

PPV: Positive predictive value, NPV: Negative predictive value

Fig.1 Size distribution report by intensity obtained from Malvern Zetasizer

\begin{tabular}{|c|c|c|c|c|c|}
\hline & & & Diam. (nm) & $\%$ Intensity & Width (nm) \\
\hline Z-Average (d.nm): & 124.1 & Peak 1: & 148.6 & 98.5 & 67.80 \\
\hline Pdl: & 0.236 & Peak 2: & 5064 & 1.5 & 571.6 \\
\hline Intercept: & 0.953 & Peak 3: & 0.000 & 0.0 & 0.000 \\
\hline
\end{tabular}

Result quality : Good

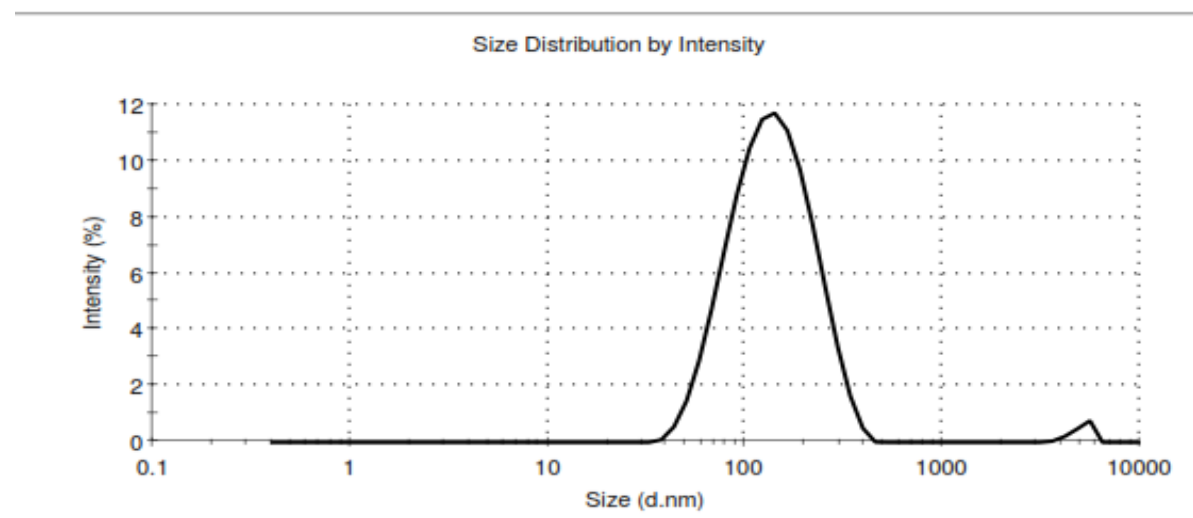

Fig.2 Zeta potential report from Malvern Zetasizer

\begin{tabular}{|c|c|c|c|c|}
\hline & & Mean $(\mathrm{mV})$ & Area $(\%)$ & Width (mV) \\
\hline Zeta Potential (mV): -23.8 & Peak 1: & -23.8 & 100.0 & 4.47 \\
\hline Zeta Deviation (mV): 4.47 & Peak 2: & 0.00 & 0.0 & 0.00 \\
\hline $\begin{array}{c}\text { Conductivity }(\mathrm{mS} / \mathrm{cm}): 0.0196 \\
\text { Result quality : Good }\end{array}$ & Peak 3: & 0.00 & 0.0 & 0.00 \\
\hline
\end{tabular}

Zeta Potential Distribution

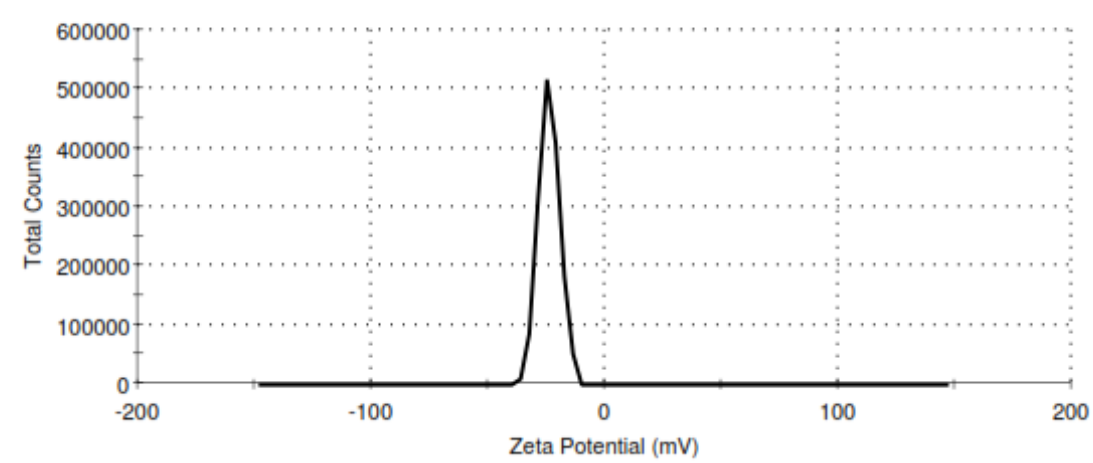


Fig.3 Scanning electron micrograph of ciprofloxacin liposomes

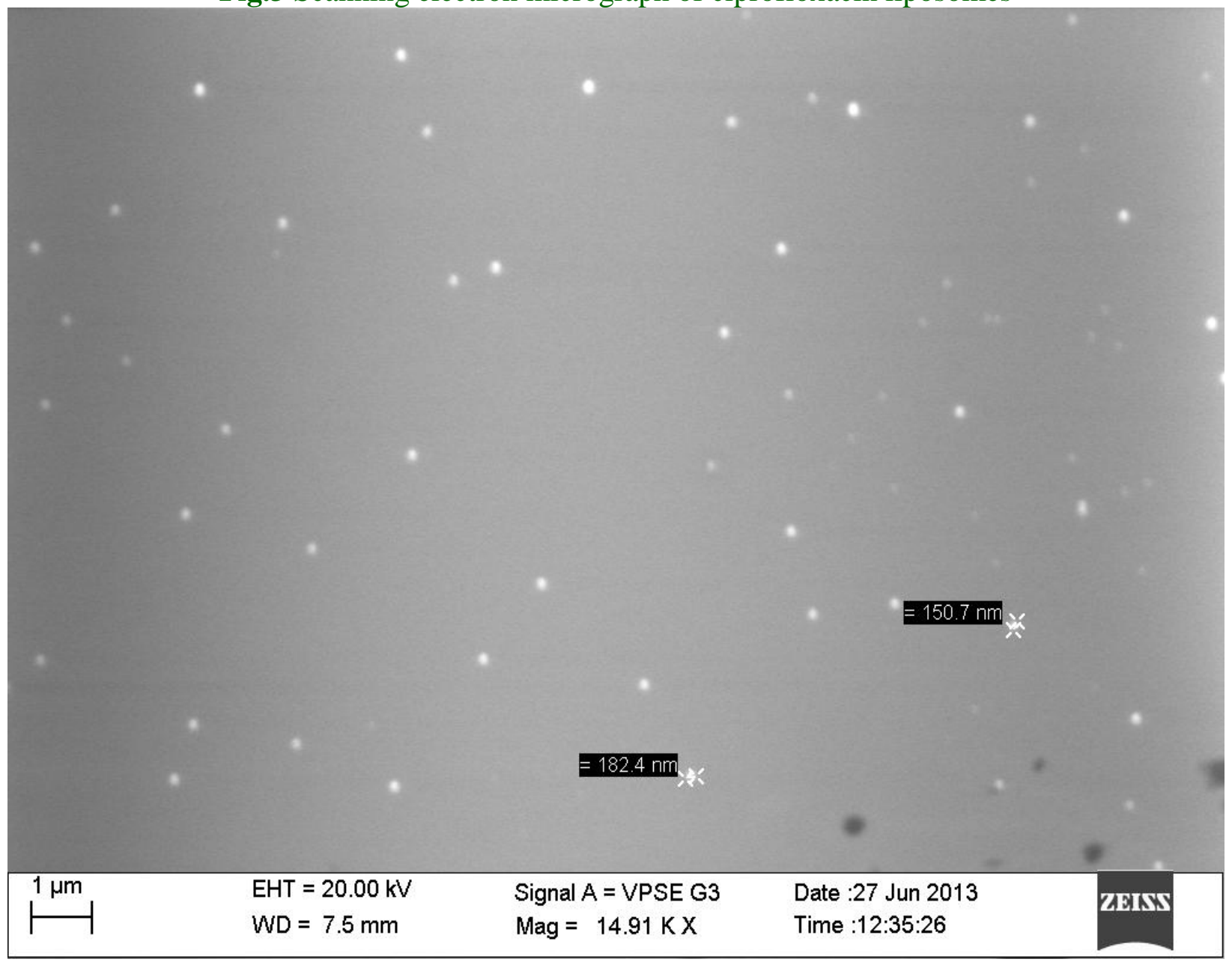

The results of validation study of FBMA and broth micro-dilution method against liposomes are given in table 3. The sensitivity, specificity, positive predictive value and negative predictive value of FBMA were found to be 100 in case of all the tested organisms. Hence FBMA is valid for liposomal MIC determination. All the four components of validity were found to vary when tested against different microorganisms. Sensitivity and negative predictive value were found to be good (equal to100) except for $S$. aureus ATTC 12600.

The fluorescent assay is a direct measurement of live cells. In case of optical density (OD) measurement, the light scattered is measured. This scattering can be done by bacteria as well as other materials present. But in fluorescence measurement the dye has to be reduced to get fluorescence and this reduction is performed by the metabolically active cells. Therefore there is less chance of getting interference by other materials. These readings are more accurate compared to the OD measurement as even with the presence of very little quantity of live cells, the fluorescence will be detected. Another advantage is that there won't be any interference by the other particles as the amount of fluorescent compound produced will be proportional to the amount of live cells. Compared to the agar dilution method the use of media can be reduced to a bigger extend by adopting this method. This method require only very little medium and reagents 
and is sensitive, rapid, economical and offers screening of large number of liposomes and opaque compounds.

\section{Acknowledgement}

This work is supported by the INSPIRE Fellowship award of Department of Science and Technology, Government of India.

\section{Conflict of interest}

The authors confirm that this article content has no conflicts of interest.

\section{References}

Alhariri, M., Azghani, A. and Omri, A. (2013) Liposomal antibiotics for the treatment of infectious diseases. Expert Opinion on Drug Delivery, 10, 15151532.

Arias, J. L. (2013) Liposomes in drug delivery: a patent review (2007). Expert opinion on therapeutic patents, 23, 1399-1414.

Bangham, A. D., Standish, M. M. and Watkins, J. C. (1965) Diffusion of univalent ions across the lamellae of swollen phospholipids. Journal of molecular biology, 13, 238-IN27.

Chattopadhyay, S. (2013) Aerosol generation using nanometer liposome suspensions for pulmonary drug delivery applications. Journal of Liposome Research, 23, 255-267.

Cheow, W. S. and Hadinoto, K. (2011) Factors affecting drug encapsulation and stability of lipid polymer hybrid nanoparticles. Colloids and Surfaces B: Biointerfaces, 85, 214-220.

Halwani, M., Mugabe, C., Azghani, A. O., Lafrenie, R. M., Kumar, A. and Omri, A. (2007) Bactericidal efficacy of liposomal aminoglycosides against Burkholderia cenocepacia. Journal of Antimicrobial Chemotherapy, 60, 760769.

Institute, C. L. S. (2012) Methods for Dilution Antimicrobial Susceptibility Tests for Bacteria That Grow Aerobically; Approved Standard - Ninth Edition. Approved standard M7-A9. Wayne, PA., Clinical and Laboratory Standards Institute.

Mugabe, C., Halwani, M., Azghani, A. O., Lafrenie, R. M. and Omri, A. (2006) Mechanism of enhanced activity of liposome-entrapped aminoglycosides against resistant strains of Pseudomonas aeruginosa. Antimicrobial agents and chemotherapy, 50, 2016-2022.

Omri, A., Ravaoarinoro, M. and Poisson, M. (1995) Incorporation, release and invitro antibacterial activity of liposomal aminoglycosides against Pseudomonas aeruginosa. Journal of Antimicrobial Chemotherapy, 36, 631-639.

Rathore, A., Jain, A., Gulbake, A., Shilpi, S., Khare, P., Jain, A. and Jain, S. K. (2011) Mannosylated liposomes bearing Amphotericin B for effective management of visceral Leishmaniasis. Journal of Liposome Research, 21, 333340.

\section{How to cite this article:}

Atulya Manuel and Nabeel Abdulrahman. 2017. Determination of Minimum Inhibitory Concentration of Liposomes: A Novel Method. Int.J.Curr.Microbiol.App.Sci. 6(8): 1140-1147. doi: https://doi.org/10.20546/ijcmas.2017.608.141 